\title{
A sensitive serodiagnostic tool for the detection of active infection of zoonotic visceral and nasopharyngeal linguatulosis
}

\author{
Marwa M. Attia ${ }^{1}$, Elshaimaa Ismael $^{2}$ and Nagla M. K. Saleh ${ }^{3}$
}

1. Department of Parasitology, Faculty of Veterinary Medicine, Cairo University, Giza 12211, Egypt; 2. Department of Veterinary Hygiene and Management, Faculty of Veterinary Medicine, Cairo University, Giza 12211, Egypt; 3. Department of Zoology, Faculty of Science, Aswan University, Aswan, Egypt.

Corresponding author: Marwa Mohamed Attia, e-mail: marwaattia.vetpara@yahoo.com

Co-authors: EI: elshaimaavet@gmail.com, NMKS: naglaakamel411@gmail.com

Received: 19-12-2018, Accepted: 10-05-2019, Published online: 24-06-2019

doi: 10.14202/vetworld.2019.883-889 How to cite this article: Attia MM, Ismael E, Saleh NMK (2019) A sensitive serodiagnostic tool for the detection of active infection of zoonotic visceral and nasopharyngeal linguatulosis, Veterinary World, 12(6): 883-889.

\begin{abstract}
Aim: This study aimed to evaluate the different serological techniques for early diagnosis of acute concurrent infections with linguatulosis in the definitive host (dogs) and an intermediate host (goats). This evaluation compared between the gold standard (GS) test (GS; examination of nasal and fecal samples in dogs and examination of lymph nodes in goats), sandwich enzyme-linked immunosorbent assay (S-ELISA), and indirect ELISA.
\end{abstract}

Materials and Methods: Fifty goats and fifty dogs were examined for the presence of Linguatula serrata nymphs and adults, respectively, besides the collection of blood samples from the examined animals for serologic testing.

Results: In goats; GS, S-ELISA, and indirect ELISA showed positivity in 32 (64\%), 28 (56\%), and 39 (78\%) samples, respectively. In dogs; GS, S-ELISA, and indirect ELISA showed positivity in $25(50 \%), 25(50 \%)$, and $30(60 \%)$ samples, respectively. S-ELISA displayed significant higher agreement with the GS test $(\geq 0.83)$ than indirect ELISA $(\leq 0.67)$ in both hosts. Infection with linguatulosis showed significant relation with the age of goats and $\operatorname{dogs}$ and the sex of goats ( $\mathrm{p}<0.05$ ).

Conclusion: S-ELISA displayed more sensitivity and specificity for the detection of concurrent infections with linguatulosis in both dogs and goats than indirect ELISA, which could detect the prior infections. Similarly, these assays could be used for diagnosis of concurrent infections with linguatulosis in human, especially the chronic ones.

Keywords: halzoun, indirect enzyme-linked immunosorbent assay, Linguatula serrata, sandwich enzyme-linked immunosorbent assay, visceral linguatulosis.

\section{Introduction}

Linguatula serrata is a cosmopolitan worldwide aberrant endoparasite of the class Pentastomida [1]. The life cycle of $L$. serrata involves definitive host (dogs, cats, foxes, and other carnivores) and intermediate hosts (herbivorous animals). Infection mechanism is by eggs ingestion; these eggs contaminate the soil and the grass through infected nasal secretions and feces.

L. serrata adults are worm-like a parasite of the upper respiratory system, including the nasal cavities, turbinate, and rarely frontal sinuses of carnivorous animals as natural definitive hosts [2]. The eggs are expelled from the respiratory passage of the final host; therefore, contaminate pastures and water resources. When the infected eggs are swallowed by suitable herbivorous animals, the larvae reach the mesenteric lymph nodes (MLNs), liver, lungs, spleen, rarely the eyes, and other organs.

Copyright: Attia, et al. Open Access. This article is distributed under the terms of the Creative Commons Attribution 4.0 International License (http://creativecommons.org/licenses/by/4.0/), which permits unrestricted use, distribution, and reproduction in any medium, provided you give appropriate credit to the original author(s) and the source, provide a link to the Creative Commons license, and indicate if changes were made. The Creative Commons Public Domain Dedication waiver (http://creativecommons.org/ publicdomain/zero/1.0/) applies to the data made available in this article, unless otherwise stated.
Different epidemiological studies were recorded in many countries throughout the world as in Egypt [2-6] and Iran [7-13]. Villedieu et al. [14] recorded infection in dogs in the United Kingdom. Different surveys were recorded in Turkey [15-17], while Gomez-Puerta et al. [18] recorded the first finding of the nymphal stage in a (vicuna) in Peru. Occurrence in dogs and livestock was recently recorded in Bangladesh and Australia [19,20].

L. serrata has zoonotic importance for human. Many cases were reported in different areas worldwide from which two patients were diagnosed with liver pentastomiasis associated with rectal adenocarcinoma [21-23]. Several cases recorded with nasopharyngeal infection or halzoun (marrara syndrome) [24-26]. Ocular linguatulosis was reported $[27,28]$, in which the L. serrata nymphs attack and damage the eyes.

Different pathological changes were detected in infected lymph nodes as softening, hemorrhages, and necrosis. Histopathologic findings revealed severe infiltration of leukocytes, macrophage, and eosinophilia with necrosis and loss of morphologic features of lymphoid follicles and its trabeculae [19,29].

The morphological features of nymphs and adult males and females of $L$. serrata were described as; tongue-shaped, flattened dorsoventrally, and 
annulated body containing about $80-90$ segments. The broad anterior end is composed of the oral opening which is subterminal and squarish, with two pairs of compound hooks surrounding this oral opening. Each body segment is covered with minute spines. The posterior part ends with the anal opening $[2,30]$.

Although different literatures described in detail the prevalence and morphology of $L$. serrata nymphs and adults; few reports described the serologic techniques used for diagnosis of linguatulosis except two reports in which one used the counter immune-electrophoresis as tool for the diagnosis [31], and the other report used the indirect enzyme-linked immunosorbent assay (ELISA) on sheep and goats in Greece [32]. Moreover, there were no reports on the diagnosis of concurrent linguatulosis using antigens prepared from nymphs and adults of $L$. serrata collected from infected goats and dogs.

This study aimed for evaluation of the potential use of $L$. serrata somatic antigens for the early detection of acute linguatulosis in goats and dogs, with a comparison between the postmortem examination (the gold standard [GS] test) and different serologic techniques (sandwich ELISA [S-ELISA] and indirect ELISA).

\section{Materials and Methods}

\section{Ethical approval}

All study procedures were approved by the Institutional Animal Care and Use of Ethical Committee of the Faculty of Veterinary Medicine, Cairo University, Egypt.

\section{Sampling and GS test}

In goats

Fifty slaughtered goats from butcher shops were inspected from March 2018 to August 2018. Blood samples for serum were collected from each animal, and MLNs were obtained and grossly examined by naked eyes for detection of $L$. serrata nymphs, according to Attia et al. [2] and Sinclair [33], and placed in warm saline solution $0.9 \%$ at $37^{\circ} \mathrm{C}$ for $20 \mathrm{~min}$. Then, the saline solution was examined under a stereoscopic microscope (LEICA M60, United State).

In dogs

Blood, fecal, and nasal swabs were collected from 50 dogs from different governorates from March 2018 to August 2018. A positive control case was recorded in the hospital of Faculty of Veterinary Medicine, Cairo University; which sneezed L. serrata adult parasites from its nasal cavity. All were dogs examined for the presence of $L$. serrata eggs in feces and nasal swabs, according to Attia et al. [2].

\section{Identification of nymphs and adult of $L$. serrata}

The collected nymphs and adults were washed in saline solution, relaxed, flattened, fixed, stained, mounted, and identified, according to Attia et al. [2] and Razavi et al. [34].

\section{Serological diagnosis}

Preparation of larval antigens

The nymphs and adults were washed several times with saline solution. Antigens were prepared from 500 nymphs and ten adults recovered from dogs. Antigens were preserved in phosphate-buffered saline (PBS) (pH 7.2) and prepared [32]. The protein content of the two antigens was measured according to Lowry et al. [35].

\section{Preparation of hyperimmune sera}

Six male rats (Rattus norvegicus albino) about $150 \mathrm{~g}$ in weight were raised for preparation of hyperimmune sera versus $L$. serrata nymphs and adults according to Innocenti et al. [36], with some modification. The rats housed in two groups (two negative controls; two immunized against $L$. serrata nymphs; and two immunized against $L$. serrata adults) in conventional rat cages. The rats were supplied with balanced rats' pellets and water ad libitum. The immunized rats were subcutaneously injected with $1 \mathrm{mg}$ protein of antigens, which mixed in $1 \mathrm{ml}$ mineral oil ( $\left(1^{\text {st }}\right.$ dose). Then, two subsequent intramuscular injections of 0.5 $\mathrm{mg}$ protein antigens mixed in the same volume of mineral oil were injected each 2 weeks interval. Rats were slaughtered for collection of blood for sera, 2 weeks after the last dose. The collected hyperimmune rats' sera were stored at $-20^{\circ} \mathrm{C}$ until used.

\section{$S$-ELISA and indirect ELISA}

All serum samples collected from the examined goats and dogs were tested with S-ELISA and indirect ELISA. Hyper-immunized rat sera against $L$. serrata nymphs and adults; negative control sera from rats and 1-month old goats and puppies, and positive control sera from naturally infected goats and dogs with $L$ serrata were used in each test.

\section{Cross-reaction of the two tests with L. serrata} antibodies

In addition to the sera from $L$. serrata cases, sera from dogs infected with Toxocara canis and Echinococcus granulosus adults were collected. Likewise, sera from goats infected with Haemonchus contortus adult and hydatid cyst were collected.

\section{Indirect ELISA}

The checkerboard titration for determination of the lowest antigen concentration and sera dilution was done, according to Harlow and Lane [37]. A ninety-six well, flat-bottomed ELISA plates were incubated overnight with $100 \mu \mathrm{l} /$ well of antigen at $20 \mu \mathrm{g} / \mathrm{ml}$ for nymphs and $10 \mu \mathrm{g} / \mathrm{ml}$ coating buffer ( $\mathrm{pH}$ 9.6) for an adult in $4^{\circ} \mathrm{C} .200 \mu \mathrm{l} /$ well of the blocking buffer (Bovine serum albumin - PBS) was added for $2 \mathrm{~h}$ at $37^{\circ} \mathrm{C}$. After 3 times washings; $100 \mu \mathrm{l}$ of diluted sera $1: 100$ in PBS were incubated at $37^{\circ} \mathrm{C}$ for $2 \mathrm{~h} .100 \mu \mathrm{l} /$ well of horseradish peroxidase anti-goat and anti-dogs IgG; anti-rat IgG conjugate (Sigma, A-5420) diluted at 1:1000 were added to each well and incubated for 
$1 \mathrm{~h}$ at room temperature. A $100 \mu \mathrm{l}$ of a substrate buffer contains (10 mg Ortho-Phenylenediamine) in citrate buffer and $30 \% \mathrm{H}_{2} \mathrm{O}_{2}$ were added to the plate wells. Finally, the ELISA plate was stopped by adding $3 \mathrm{~N}$ $\mathrm{H}_{2} \mathrm{SO}_{4}$, and the absorbance values or optical density (OD) were obtained from an ELISA reader (Bio-Rad, USA) at $450 \mathrm{~nm}$. The sera were considered to be positive when the absorbance values were as or more than the cut off value equal to a double fold of the mean negative sera; the ELISA was done, according to Kouam et al. [32].

\section{S-ELISA}

Checkerboard titration was done according to Harlow and Lane [37] to obtain the different dilution of antigen, sera, and conjugate. A ninety-six well, flat-bottomed ELISA plates were incubated overnight with $100 \mu \mathrm{l} /$ well of hyperimmune sera from adult and nymphs diluted in carbonate buffer. After 3 times washings with PBS- Tween-20; $100 \mu \mathrm{l}$ of diluted antigens of nymphs and adults at $10 \mu \mathrm{g} / \mathrm{ml}$ in PBS were incubated $37^{\circ} \mathrm{C}$ for $2 \mathrm{~h} .100 \mu \mathrm{l} /$ well of naturally infected and unknown sera were added with concentration 1:100 in PBS. Then, run the ELISA plate as indirect ELISA procedure mentioned above. The procedure followed according to Ana and Finlay [38], Rui et al. [39], and Dixit et al. [40].

\section{Statistical analysis}

The cutoff point for a positive result was taken as $2 \times$ mean of OD of negative control sera [41]. To evaluate the values of sensitivity, specificity, accuracy, positive predictive values (PPV), and negative predictive values (NPV) and other points of screening diagnostic, individual screening tests were performed in ELISA, S-ELISA, and GS tests. The GS was considered the most sensitive and was assigned as a benchmark test.

Statistical analysis of agreement between ELISA, S-ELISA, and GS tests was performed using the Kappa agreement test. The Landis and Koch scale [42] was used to measure the degree of agreement according to the Kappa value, with the scores divided into: $<0$ no agreement; $0.0-0.20$ slight; 0.21 0.40 fair; 0.41-0.60 moderate; 0.61-0.80 substantial; 0.81-0.99 almost perfect; and 1 perfect. Pearson correlation was estimated between ELISA, S-ELISA, and GS tests. Differences between groups were assessed by Chi-square test. Values of $p \leq 0.05$ were considered statistically significant. All statistical inference was performed on PASW Statistics, Version 18.0. software (SPSS Inc., Chicago, IL, USA).

\section{Results and Discussion}

\section{Identification of the parasites}

The nymphs, adults, and its eggs identified for L. serrata as recorded by Attia et al. [2] (Figure-1).

\section{Characterization of antigens}

The $L$. serrata crude extract antigens were found to contain 20 and $30 \mu \mathrm{g} / \mathrm{ml}$ of total protein in nymphs and adults, respectively.

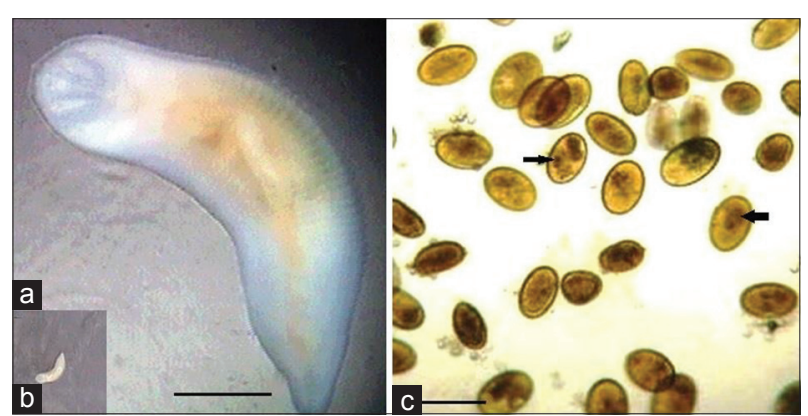

Figure-1: Adult of Linguatula serrata ( $a$ and $b$ ) showing the anterior end of the adult with four hooks surround mouth openings. (b) The eggs of $L$. serrata from nasal swabs showing the claw of the larvae pointed by arrows. Scale bar in (a) $1 \mathrm{~mm}$, while in (c) $100 \mu \mathrm{m}$.

\section{Checker board titration}

In indirect ELISA; the concentration of the antigens were $20 \mu \mathrm{g}$ and $10 \mu \mathrm{g} / \mathrm{ml}$ for nymphs and adult respectively, and the diluted serum was 1:100. While in S- ELISA; the concentration of the antigens were $10 \mu \mathrm{g} / \mathrm{ml}$ for nymphs and adults, and the diluted serum was 1:100, while the conjugate for both tests was $1: 1000$.

\section{Cross-reaction of the two tests with $L$. serrata antibodies}

Crude antigens of $T$. canis adult and E. granulosus adults, $H$. contortus adult, and E. granulosus hydatid cyst scolices were used and processed for specificity analyses. There was no cross-reaction recorded in the two tests, all the OD values for those crude antigens positive sera were $<0.25$, whereas the OD value of control negative sera of rats was 0.12 and of dogs was 0.23 while in goats were 0.20 .

\section{OD of positive control sera from dogs and goats}

The cutoff values were 0.51 and 0.56 for dogs' sera and 0.51 and 0.50 for goats' sera while using indirect ELISA and S-ELISA, respectively. Positive OD values for dogs ranged between 0.5 and 1.0 in indirect ELISA while in S-ELISA the OD values were 0.61.9. The OD values in goats were $0.45-1.8$ in indirect ELISA while in S-ELISA the OD values in positive cases recorded 0.5-1.8.

\section{Positive indirect ELISA, S-ELISA, and GS tests in goats}

Examination of lymph nodes in goats (GS) showed positivity in 32 samples (64\%). The indirect ELISA test of goats' sera showed seropositivity in 39 samples $(78 \%)$ while the S-ELISA showed seropositivity in 28 samples (56\%) (Table-1).

The indirect ELISA test of goats' sera showed a sensitivity of $100 \%$, and specificity of $61.11 \%$, with an accuracy of $86 \%$ while the S- ELISA test showed sensitivity of $87.5 \%$, and specificity of $100 \%$, with an accuracy of $92 \%$ (Table-2).

For the agreement analysis, the Kappa values obtained were significantly substantial between ELISA and GS tests $(\mathrm{K}=0.67 ; \mathrm{p}=0.000)$ and between S-ELISA and GS $(\mathrm{K}=0.83 ; \mathrm{p}=0.000)$, while it was 
significantly moderate between ELISA and S-ELISA $(\mathrm{K}=0.53 ; \mathrm{p}=0.000)$ (Table- 3 ).

Using the Pearson's test for correlation, a significant positive correlation was proved between the number of nymphs detected by GS test and the optical densities obtained from indirect ELISA and S-ELISA tests $(\mathrm{r}=0.85$ and $\mathrm{r}=0.90 ; \mathrm{p}<0.000$; respectively) (Table-4).

\section{Positive indirect ELISA, S-ELISA, and GS tests in dogs}

The examination of feces in dogs (GS) showed positivity in 25 samples $(50 \%)$. The indirect ELISA test of dogs' sera showed seropositivity in 30 samples $(60 \%)$, while the S-ELISA showed seropositivity in 25 samples (50\%) (Table-1).

The indirect ELISA test of dogs' sera showed sensitivity of $84 \%$ and specificity of $64 \%$, with an accuracy of $74 \%$, while the S-ELISA test showed

Table-1: ELISA, S-ELISA, and GS result in samples from goats and dogs, Cairo, Egypt, 2018 ( $n=50$ goats; 50 dogs).

\begin{tabular}{lccc}
\hline $\begin{array}{l}\text { Diagnostic } \\
\text { test }\end{array}$ & GS (\%) & S-ELISA (\%) & $\begin{array}{c}\text { Indirect } \\
\text { ELISA (\%) }\end{array}$ \\
\hline Goats & $32(64)$ & $28(56)$ & $39(78)$ \\
Positive & $22(44)$ & $11(22)$ \\
$\quad$ Negative & $18(36)$ & & \\
Dogs & & $25(50)$ & $30(60)$ \\
Positive & $25(50)$ & $25(50)$ & $20(40)$ \\
Negative & $25(50)$ & 25 \\
\hline
\end{tabular}

ELISA=Enzyme-linked immunosorbent assay,

S-ELISA = Sandwich enzyme-linked immunosorbent assay, GS=Gold standard (examination of lymph nodes in goats and examination of feces in dogs) sensitivity of $96 \%$ and specificity of $96 \%$, with an accuracy of $96 \%$ (Table-2).

For the agreement analysis, the Kappa value obtained was significantly moderate between ELISA and $\mathrm{GS}$ tests $(\mathrm{K}=0.48 ; \mathrm{p}=0.001)$, and between ELISA and S-ELISA $(\mathrm{K}=0.48 ; \mathrm{p}=0.001)$, while it was significantly almost perfect between S-ELISA and GS $(\mathrm{K}=0.92 ; \mathrm{p}=0.000)($ Table- 3$)$.

\section{Frequency of infection in goats and dogs}

In goats, the frequency of $L$. serrata infection was related to the sex of the animal and was significantly higher in female goats than in males ( $\mathrm{p}=0.006$ ). However, in dogs, the frequency of infection was not statistically related to the sex of the animal $(p>0.05)$ (Figure-2, Table-5).

In goats, the frequency of $L$. serrata infection was related to the age of the animal and was significantly higher in goats aged 3 years or more $(\mathrm{p}=0.000)$. In addition, in dogs, it was statistically related to the age of the animal. Dogs of 1 year old and 5 years old showed the highest frequencies of infection $(\mathrm{p}=0.000)$, (Figure-3 and Table-5).

In goats, the GS test revealed $64 \%$ positivity, while S-ELISA revealed $56 \%$ positivity in comparison to indirect ELISA which revealed $78 \%$ positivity; this is due to that S-ELISA could detect the concurrent infection, while indirect ELISA could detect the antibody levels of the previous infection from 6 months ago. Positive S-ELISA results were higher than GS, which may be due to the presence of infection in different organs other than MLNs.

Table-2: Screening test results for indirect ELISA and S-ELISA in a sample from goats and dogs, Cairo, Egypt, 2018.

\begin{tabular}{|c|c|c|c|c|}
\hline \multirow[t]{2}{*}{ Screening test } & \multicolumn{2}{|c|}{ Goats } & \multicolumn{2}{|c|}{ Dogs } \\
\hline & Indirect-ELISA (\%) & S-ELISA (\%) & Indirect-ELISA (\%) & S-ELISA (\%) \\
\hline Sensitivity & 100 & 87.5 & 84 & 96 \\
\hline Specificity & 61.11 & 100 & 64 & 96 \\
\hline Type 1 error (false positive) & 38.89 & 0 & 36 & 4 \\
\hline Type 2 error (false negative) & 0 & 12.5 & 16 & 4 \\
\hline Apparent prevalence & 78 & 56 & 60 & 50 \\
\hline PPV & 82.05 & 100 & 70 & 96 \\
\hline NPV & 100 & 81.82 & 80 & 96 \\
\hline Accuracy & 86 & 92 & 74 & 96 \\
\hline Likelihood ratio positive & 2.57 & - & 2.33 & 24 \\
\hline Likelihood ratio negative & 0 & 0.125 & 0.25 & 0.04 \\
\hline
\end{tabular}

Indirect-ELISA=Indirect enzyme-linked immunosorbent assay, S-ELISA=Sandwich enzyme-linked immunosorbent assay, $\mathrm{PPV}=$ Positive predictive value, NPV=Negative predictive value, GS=Gold standard; True prevalence determined by GS was $64 \%$ in goats and $50 \%$ in dogs

Table-3: Agreement between indirect ELISA, S-ELISA, and GS in samples from goats and dogs, Cairo, Egypt, 2018.

\begin{tabular}{llclr}
\hline Animal & Diagnostic test & Kappa value & Agreement & p-value \\
\hline Goats & Indirect-ELISA versus GS & 0.67 & Substantial & 0.000 \\
& S-ELISA versus GS & 0.83 & Substantial & 0.000 \\
& Indirect-ELISA versus S-ELISA & 0.53 & Moderate & 0.000 \\
Dogs & Indirect-ELISA versus GS & 0.48 & Moderate & 0.001 \\
& S-ELISA versus GS & 0.92 & Almost perfect & 0.000 \\
& Indirect-ELISA versus S-ELISA & 0.48 & Moderate & 0.001 \\
\hline
\end{tabular}

Indirect-ELISA=Indirect enzyme-linked immunosorbent assay, S-ELISA=Sandwich enzyme-linked immunosorbent assay, $\mathrm{GS}=$ Gold standard (examination of lymph nodes in goats and examination of feces in dogs) 
Table-4: Correlation between indirect ELISA, S-ELISA, and GS in samples from goats and dogs, Cairo, Egypt, 2018.

\begin{tabular}{llcc}
\hline Animal & Diagnostic test & $\begin{array}{c}\text { Pearson } \\
\text { correlation }\end{array}$ & p-value \\
\hline Goats & Indirect-ELISA versus GS & 0.85 & 0.000 \\
& S-ELISA versus GS & 0.90 & 0.000 \\
& Indirect-ELISA versus & 0.93 & 0.000 \\
& S-ELISA & & \\
Dogs & Indirect-ELISA versus GS & - & - \\
& S-ELISA versus GS & - & - \\
& Indirect-ELISA versus & 0.69 & 0.000 \\
& S-ELISA & & \\
\hline
\end{tabular}

Indirect-ELISA = Indirect enzyme-linked immunosorbent assay, S-ELISA=Sandwich enzyme-linked immunosorbent assay, GS=Gold standard (examination of lymph nodes in goats and examination of feces in dogs)

Table-5: Frequency of $L$. serrata infection in goats and dogs in relation to sex and age of animal, Cairo, Egypt, 2018.

\begin{tabular}{lcc}
\hline Characteristics & Goat & Dog \\
\hline Sex & & \\
Female & $16 / 18(88.89 \%)$ & $9 / 20(45 \%)$ \\
Male & $16 / 32(50 \%)$ & $16 / 30(53.33 \%)$ \\
p-value & $0.006 *$ & 0.387 \\
Age (years) & & \\
1 & $1 / 9(11.11 \%)$ & $6 / 6(100 \%)$ \\
2 & $9 / 18(50 \%)$ & $7 / 23(30.43)$ \\
3 & $13 / 13(100 \%)$ & $6 / 13(46.15 \%)$ \\
4 & $4 / 5(80 \%)$ & $0 / 2(0 \%)$ \\
5 & $5 / 5(100 \%)$ & $6 / 6(100 \%)$ \\
p-value & $0.000 *$ & $0.000 *$ \\
\hline
\end{tabular}

L. serrata $=$ Linguatula serrata. ${ }^{*}$ Indicate significance at $\mathrm{p}<0.05$

From these comparisons, it was revealed that the sensitivity of the two tests was similar in goats while high in S-ELISA in dogs. The specificity of S-ELISA was high in dogs and goats.

This sensitive S-ELISA improves and simplifies the detection of linguatulosis in animals.

Pentastomiasis has a zoonotic potential to human, which is difficult to be diagnosed or might be misdiagnosed with other chronic diseases such as malignant tumors, cysticercosis, and lung diseases [43].

Advanced technique as polymerase chain reaction test was previously used for identification but had not been evaluated for diagnosis of linguatulosis [44]. For this reason, this work developed a new sensitive technique for diagnosis of concurrent infection of one of the pentastomes ( $L$. serrata), and we used two animals; the definitive host (dogs) and an intermediate host (goats) that represented the most grazing animal around the dogs, to be a good example for human testing.

Using the crude extract of $L$. serrata antigens was presented a reliable, non-invasive diagnostic tool for detecting active infections. The sensitivity and specificity of any immunoassay are dependent on the antigen capture efficiency of an antibody which is subsequently governed by the nature of antibody. This

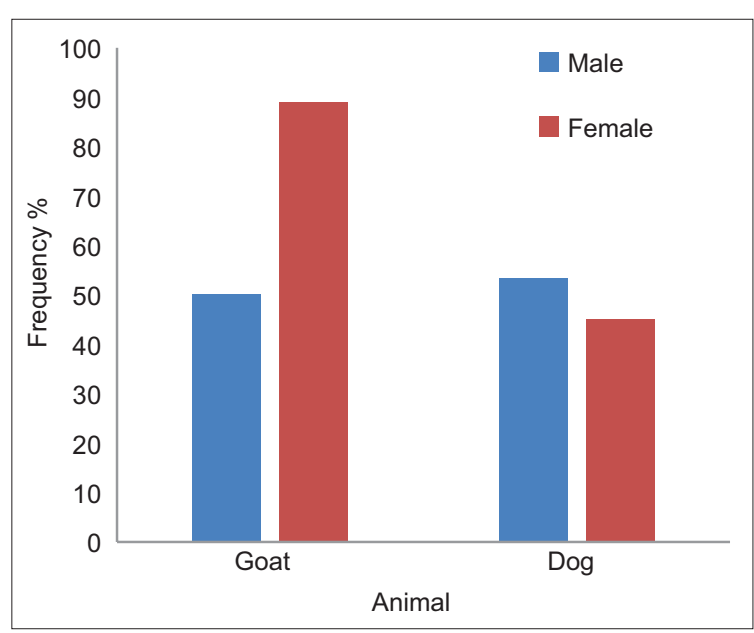

Figure-2: Frequency of Linguatula serrata infection in goats and dogs in relation to sex of the animal, Cairo, Egypt, 2018.

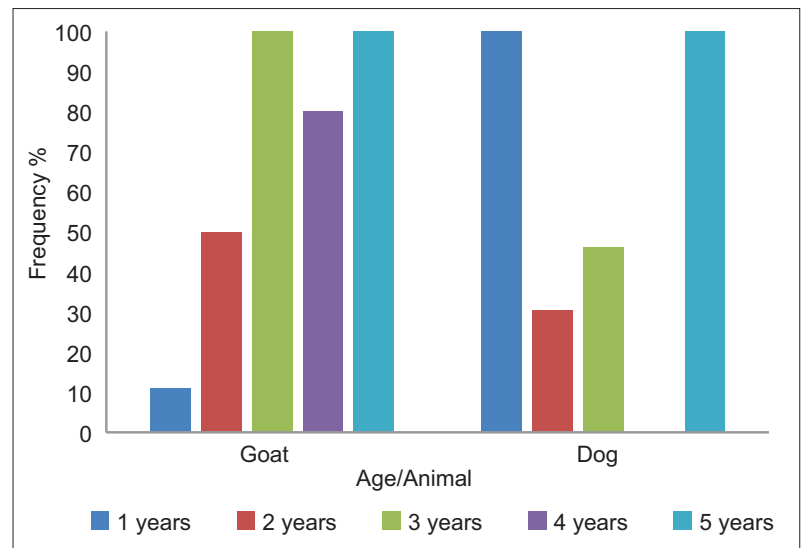

Figure-3: Frequency of Linguatula serrata infection in goats and dogs in relation to the age of the animal, Cairo, Egypt, 2018.

led to the different assay sensitivities of the commercially available kits using different types of antibodies which could be monoclonal or polyclonal at the capture stage in ELISA.

Considering linguatulosis importance in human and animals, it seems necessary to do more investigations in both domestic and wild carnivores and herbivores. Only a few reports described the seroprevalence and evaluated the indirect ELISA for 121 sheep sera and determined its sensitivity, specificity, PPV, NPV, and accuracy of the somatic antigens, which were $90.4 \%, 75.0 \%, 84.6 \%, 83.7 \%$, and $84.2 \%$, and those for excretory-secretory antigen were $89.0 \%$, $93.8 \%, 95.6 \%, 84.9 \%$, and $90.9 \%$, respectively [44]. However, indirect ELISA and S-ELISA in our study showed higher values than that report. Another report only used indirect ELISA for seroprevalence [32].

Other serological tests were used as counter immunoelectrophoresis used for diagnosis of linguatulosis in sheep [31], but that was a qualitative serological test, while the two tests used in this study were quantitative tests with high sensitivity and specificity and can be used for field studies or for human cases 
suffering from chronic disease, which could infect several organs.

\section{Conclusion}

In this study, we differentiated between two assays to distinguish the sensitivity and specificity of each test. S-ELISA showed higher sensitivity and specificity than indirect ELISA, so, it can be applied in the field studies or laboratories for diagnosing of linguatulosis because it detected the concurrent circulating antigens. S-ELISA could be considered as a new serological technique for the diagnosis of linguatulosis in goats and dogs. Moreover, S-ELISA could be used in the diagnosis of linguatulosis in human with halzoun or visceral form.

\section{Authors' Contributions}

MMA, EI, and NMKS conceived and designed the experiments. MMA and NMKS performed the experiments. EI analyzed and interpreted the data. All authors participated in draft and revision of the manuscript. All author have read and approved the final manuscript.

\section{Acknowledgments}

All authors are thankful to the staff members of Cairo abattoir for their help in the collection of samples. The authors did not receive any external funding for this study.

\section{Competing Interests}

The authors declare that they have no competing interests.

\section{Publisher's Note}

Veterinary World remains neutral with regard to jurisdictional claims in published institutional affiliation.

\section{References}

1. Hendrix, C.M. and Robinson, E. (2006) Diagnostic Parasitology for Veterinary Technicians. $3^{\text {rd }}$ ed. Mosby Elsevier, Philadelphia, PA, USA

2. Attia, M.M., Mahdy, O.A. and Saleh, N.M.K. (2017) Prevalence of Linguatula serrata (Order: Pentastomida) nymphs parasitizing camels and goats with experimental infestation of dogs in Egypt. Int. J. Adv. Res. Biol. Sci., 4(8): 197-205.

3. Khalil, M.G. (1970) Incidence of Linguatula serrata infection in Cairo mongrel dogs. J. Parasitol., 56(3): 485.

4. Khalil, M.G. (1972) Linguatula serrata (Pentastomida) parasitizing humans and animals in Egypt, neighboring countries and elsewhere: A review. J. Egypt. Public Health Assoc., 47(6): 363-369.

5. Khalil, M.G. (1973) Linguatula serrata from mongrel dogs in El-Dakhla oasis (Egypt). J. Parasitol., 59(2): 288.

6. Khalil, M.G. (1976) Prevalence of Linguatula serrata infection in animals from the Cairo abattoir. J. Parasitol., 62(1): 126

7. Meshgi, B. and Asgarian, O. (2003) Prevalence of Linguatula serrata infestation in stray dogs of Shahrekord, Iran. J. Vet. Med. B Infect. Dis. Vet. Public Health, 50(9): 466-467.

8. Shekarforoush, S.S., Razavi, S.M. and Izadi, M. (2004)
Prevalence of Linguatula serrata nymphs in sheep in Shiraz, Iran. Small Rumin. Res., 52(1): 99-101.

9. Tavassoli, M., Tajic, H., Dalir-Naghadeh, B. and Hariri, F. (2007) Prevalence of Linguatula serrata nymphs and gross changes of infected mesenteric lymph nodes in sheep in Urmia, Iran. Small Rumin. Res., 72(1): 73-76.

10. Oryan, A., Sadjjadi, S.M., Mehrabani, D. and Rezaei, M. (2008) The status of Linguatula serrata infection of stray dogs in Shiraz, Iran. Comp. Clin. Path., 17(1): 55-60.

11. Nourollahi, F.R., Kheirandish, R., Norouzi, E.A. and Fathi, S. (2010) The prevalence of Linguatula serrata nymphs in goats slaughtered in Kerman slaughterhouse, Kerman, Iran. Vet. Parasitol., 171(1-2): 176-178.

12. Rezaei, H., Ashrafihelan, J., Nematollahi, A. and Mostafavi, E. (2012) The prevalence of Linguatula serrata nymphs in goats slaughtered in Tabriz, Iran. J. Parasit. Dis., 36(2): 200-202.

13. Hajipour, N., Tavassoli, M., Eslamlo, A.A. and Javadi, S.H. (2018) Investigation of histopathological changes caused by adult stage of Linguatula serrata in dog. Comp. Clin. Path., 27(2): 717-720.

14. Villedieu, E., Sanchez, R., Jepson, R. and Haar, G.T. (2017) Nasal infestation by Linguatula serrata in a dog in the UK: A case report. J. Small Anim. Pract., 58(3): 183-186.

15. Aldemir, O.S. (2004a) The distribution of Linguatula serrata in stray dogs in the Erzurum province. Turkiye Parazitol. Derg., 28(1): 42-44.

16. Aldemir, O.S. (2004b) Prevalence of Linguatula serrata (Frohlich, 1789) nymphs in sheep in the province of Kars, Turkey. J. Fac. Vet. Med. Univ. Kafkas Kars (Turkey), 10(2): 135-137.

17. Aldemir, O.S., Aydenizoz, M. and Atesoglu, O. (2014) Parasitological and pathological investigations on Linguatula serrata nymphs in mesenteric lymph nodes in sheep in Konya region in Turkey. Turk. J. Agric. Food Sci. Technol, 2(5): 224-227.

18. Gomez-Puerta, L.A., Pachecob, J.I., Angulo-Tisoc, J.M., Lopez-Urbina, M.T. and Gonzalez, A.E. (2017) First finding of nymphal stages of Linguatula serrata in a South American camelid, a vicuña from Peru. Vet. Parasitol.,15 (244): 21-24.

19. Islam, R., Anisuzzaman, M., Hossain, S., Alama, Z., Islam, A., Khan, A.H.N., Kabir, E., Hatta, T., Alim, A. and Tsuji, N. (2018) Linguatula serrata, a food-borne zoonotic parasite, in livestock in Bangladesh: Some pathologic and epidemiologic aspects. Vet. Parasitol., 13: 135-140.

20. Shamsi, S., McSpadden, K., Baker, S. and Jenkins, D.J. (2017) Occurrence of tongue worm, Linguatula serrata (Pentastomida: Linguatulidae) in wild canids and livestock in South Eastern Australia. Int. J. Parasitol., 6(3): 271-277.

21. Bygbjerg, I.C. and Rask, M.P. (1978) Pentastomiasis and cancer of the colon. Trans. R. Soc. Trop. Med. Hyg., 72(1): 54-55.

22. Maleky, F. (2001) A case report of Linguatula serrata in human throat from Tehran, central Iran. Ind. J. Med. Sci., 55(8): 439-441.

23. Yazdani, R., Sharifi, I., Bamorovat, M. and Mohammadi, M.A. (2014) Human linguatulosis caused by Linguatula serrata in the city of Kerman, South Eastern Iran case report. Iran. J. Parasitol., 9(2): 282-285.

24. Papadakis, A.M. and Hourmouziadis, A.N. (1958) Human infestation with Linguatula serrata: Report of a case. Trans. R. Soc. Trop. Med. Hyg., 52(5): 454-455.

25. Morsy, T.A., El-Sharkawy, I.M. and Lashin, A.H. (1999) Human nasopharyngeal linguatuliasis (Pentasomida) caused by Linguatula serrata. J. Egypt. Soc. Parasitol., 29(3): 787-790.

26. Yagi, H., El Bahari, S., Mohamed, H.A., El Ahmed, R.S., Mustafa, B., Mahmoud, M., Saad, M.B., Sulaiman, S.M. and El Hassan, A.M. (1996) The Marrara syndrome: A hypersensitivity reaction of the upper respiratory tract and buccopharyngeal mucosa to nymphs of Linguatula serrata. 
Acta Trop., 62(3): 127-134.

27. Lazo, R.F., Hidalgo, E., Lazo, J.E., Bermeo, A., Liaguno, M., Murillo, J., Teixeira, V.P. (1999) Ocular linguatuliasis in Ecuador: Case report and morphometric study of the larva of Linguatula serrata. Am. J. Trop. Med. Hyg., 60(3): 405-409.

28. Koehsler, M., Walochnik, J., Georpopoulos, M., Pruente, C., Boeckeler, W., Auer, H., and Barisani-Asenbauer, T. (2011) Linguatula serrata tongue worm in human eye, Austria. Emerg. Infect. Dis., 17(5): 870-872.

29. Miclaus, V., Mihalca, A.D., Negrea, O. and Oana, L. (2008) Histological evidence for inoculative action of immature Linguatula serrata in lymph nodes of intermediate host. Parasitol. Res., 102(6): 1385-1387.

30. Banaja, A.A. (1983) Scanning electron microscopy examination of larval Linguatula serrata Frolich (Linguatulidae: Pentastomida). Z. Parasitenkd., 69(2): 271-277.

31. Yektaseresht, A., Asadpour, M., Jafari, A. and Malekpour, S.H. (2017) Seroprevalence of Linguatula serrata infection among sheep in Fars province, south of Iran. J. Zoonotic Dis., 2(2): 45-50.

32. Kouam, M.K., Diakou, A., Kantzoura, V., Feidas, H., Theodoropoulou, H. and Theodoropoulos, G. (2014) An analysis of seroprevalence and risk factors for parasitic infections of economic importance in small ruminants in Greece. Vet. J., 202(1): 146-52.

33. Sinclair, K.B. (1954) The incidence and life cycle of Linguatula serrata (Frohlich 1789) in Great Britain. J. Comp. Pathol., 64(4): 371-383.

34. Razavi, S.M., Shekarforoush, S.S. and Izadi, M. (2004) Prevalence of Linguatula serrata nymphs in goats in Shiraz, Iran. Small Rumin. Res., 54(3): 213-217.

35. Lowry, O.H., Rosenbrough, N.J. and Randall, R.J. (1951) Protein measurement with Folin phenol reagent. J. Biol. Chem., 193(1): 265-275.

36. Innocenti, L., Masetti, M., Macchioni, G. and Giorgi, F.
(1995) Larval salivary gland proteins of the sheep nasal botfly, (Oestrus ovis L.) are major immunogens in infested sheep. Vet. Parasitol., 60(3-4): 273-282.

37. Harlow, E. and Lane, D. (1988) Antibodies. A Laboratory Manual. Cold Spring Harbor Laboratory, Cold Spring Harbor, New York.

38. Ana, M.E., Finlay, C.M. (1994) Sandwich enzyme-linked immunosorbent assay for detection of excretory-secretory antigens in humans with fascioliasis. J. Clin. Microbiol., 32(1): 190-193.

39. Rui, C., Lu, S., Lou, D., Lin, A., Zeng, X., Ding, Z., Wen, L., Ohta, N., Wang, J. and Fu, C. (2008) Evaluation of a rapid ELISA technique for detection of circulating antigens of Toxoplasma gondii. Microbiol. Immunol., 52(3): 180-187.

40. Dixit, C.K., Vashist, S.K., O'Neill, F.T., O'Reilly, B., MacCraith, B.D. and O'Kennedy, R. (2010) Development of a high sensitivity rapid sandwich ELISA procedure and its comparison with the conventional approach. Anal. Chem., 82(16): 7049-7052.

41. Romero, J.A., Arias, M.S., Suarez, J.L., Paz-Silva, A., Francisco, I., Alonso, F., Cortiñas, F.J., Dacal, V., Romasanta, A., Morrondo, P., Díez-Baños, P., Scala, A. and Sánchez-Andrade, R. (2010) Application of the analysis of serum antibodies (immunoglobulins $\mathrm{M}$ and $\mathrm{G}$ ) to estimate the seroprevalence of ovine oestrosis and to evaluate the effect of chemotherapy. J. Med. Entomol., 47(3): 477-481.

42. Landis, J.R. and Koch, G.G. (1977) The measurement of observer agreement for categorical data. Biometrics, 33(1): 159-174.

43. Vanhecke, C., Le-Gall, P., Le Breton, M. and Malvy, D. (2016) Human pentastomiasis in Sub-Saharan Africa. Med. Mal. Infect., 46(6): 269-275.

44. Gjerde, B. (2013) Phylogenetic position of Linguatula arctica and Linguatula serrata (Pentastomida) as inferred from the nuclear $18 \mathrm{~S}$ rRNA gene and the mitochondrial cytochrome c oxidase subunit I gene. Parasitol. Res., 112(10): 3517-3525. 